\title{
Fixation of Roux Limb and Duodenal Stump Effects the Prevention of Peterson's Internal Hernia and Roux Stasis Syndrome After Laparoscopic Distal Gastrectomy
}

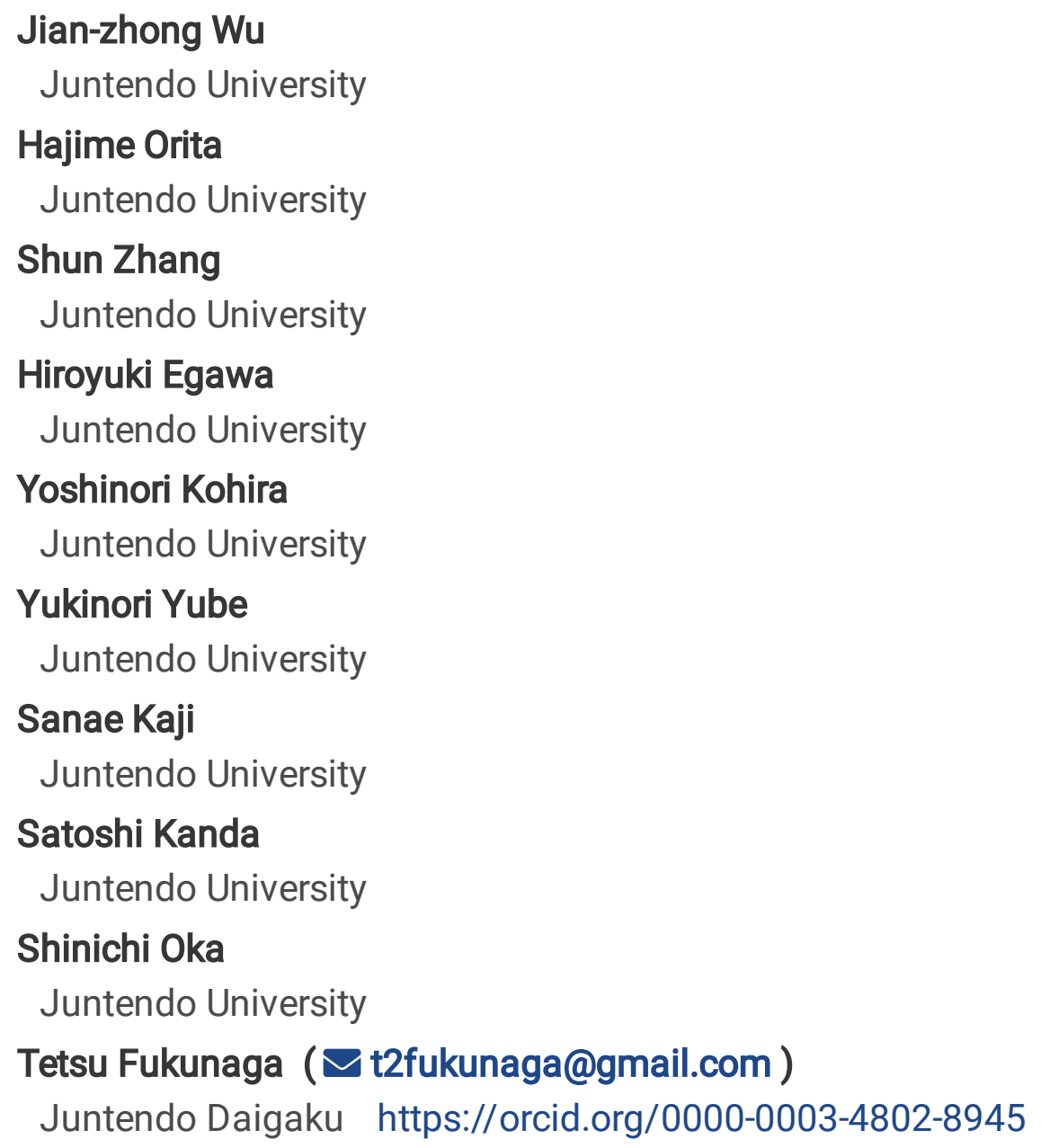

Research article

Keywords: Laparoscopy distal gastrectomy , Roux-en-Y reconstruction, Internal hernia, Roux limb syndrome, Gastric cancer

Posted Date: October 31st, 2019

DOI: https://doi.org/10.21203/rs.2.16629/v1 
License: (c) (i) This work is licensed under a Creative Commons Attribution 4.0 International License. Read Full License 


\section{Abstract}

Background Roux-en-Y (R-Y) reconstruction is a standard option for reconstruction after laparoscopic distal gastrectomy (LDG). Though this technique takes more time than Billroth I (B-I) reconstruction, leakage is rare, and it is useful for management of a small remnant stomach. Complications occur and include Petersen's hernia and Roux stasis syndrome. Petersen's hernia is a rare complication that results in significant morbidity and poor quality of life.Here we report a case series of a simple and effective method for preventing Petersen's hernia and Roux stasis syndrome.

Methods We performed ante-colic R-Y reconstruction after LDG. After R-Y reconstruction, we fixed the alimentary limb onto the duodenal stump in a proper radian. Via this small improvement in the Roux limb, the alimentary limb was placed to the right of the ligament of Treitz. This not only changed the anatomy of the Petersen's defect, but it also kept a fluent direction of gastrointestinal anastomosis and avoided a cross-angle after jejunojejunostomy.

Results From July 2015 to March 2017, we performed this technique in 31 consecutive patients. The operative time was (308.0 \pm 84.6 minutes). Fixation of the alimentary limb onto the duodenal stump took about 10 minutes. Two (6.5\%) patients experienced pneumonia and pancreatitis, respectively. No patient required reoperation or readmission. All patients were followed up for at least 2 years, and none of the patients developed postoperative complications related to internal hernia or Roux stasis syndrome.

Conclusions This 10 minute technique is a very effective method to reduce the onset of Petersen's hernia and Roux stasis syndrome in patients who undergo LDG.

\section{Introduction}

$\mathrm{R}-\mathrm{Y}$ and $\mathrm{B}-\mathrm{Q}$ reconstruction are the preferred reconstruction techniques after LDG for gastric cancer in Eastern countries, while $\mathrm{R}-\mathrm{Y}$ and Billroth $\otimes(\mathrm{B}-\mathrm{Z})$ reconstruction are more prevalent choices in Western countries[1]. Though $\mathrm{R}-\mathrm{Y}$ reconstruction is more time intensive than either $\mathrm{B}-\mathrm{\bigotimes}$ or $\mathrm{B}-\mathrm{\nabla}$ reconstruction (because of the relatively complex R-Y procedure and the additional anastomosis), leakage is rare and it is useful when there is a small remnant stomach. $R-Y$ reconstruction is advantageous over B-I reconstruction in anastomotic leakage because there is no anastomotic tension and the blood supply is preserved. [2.3.4]. Moreover, R-Y reconstruction reduces the incidence of bile reflux that is associated with

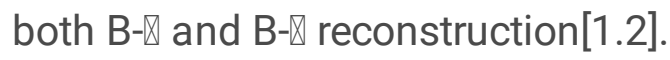

There are two anatomical routes for R-Y reconstruction-antecolic and retrocolic. Some surgeons strongly recommend the antecolic approach, which can provide better exposure to the mesenteric defect and lesser mesenteric defects than the retrocolic approach [5.6.7.8]. Although R-Y reconstruction is favored over B-I reconstruction, two mesenteric defects are created during an antecolic R-Y reconstruction procedure: jejunojejunostomy and Petersen's defect-both of which may lead to internal hernia.Peterson's hernia is located at Petersen's defect, which is the gap between the Roux limb and the transverse mesocolon [6]. It is a rare complication that result in significant morbidity and poor quality of life. 
However, meticulous closure of the jejunojejunostomy defect completely eliminates hernias in this location[8]. Thus, with an ante-colic R-Y reconstruction procedure following LDG, the primary difficulty is how to prevent Petersen's hernia.

In addition, R-Y reconstruction following LDG is nearly universally regarded as an effective method to prevent postoperative bile reflux or the development of gastric remnant cancer[9.10]. However, some patients may develop Roux stasis syndrome, which is related to a functional obstruction of the Roux limb and is characterized by abdominal pain, vomiting and post-prandial nausea[11]. The pathogenic mechanisms of Roux stasis syndrome are not completely identified, though the angle of the Roux limb may be a cause[12].

Given that surgical technical factors involving the Roux limb play an important role in the development of these two complications, we developed a method to narrow the Petersen's defect and reduce Roux stasis syndrome. Herein we report the clinical outcomes of our technique.

\section{Patients And Methods}

\section{Patients:}

Between July 2015 and March 2017, 31 patients with gastric cancer underwent LDG with ante-colic R-Y reconstruction by the same surgeon at the Department of Gastroenterology and Minimally Invasive Surgery at Juntendo University School of Medicine. Clinical parameters included clinicopathologic characteristics, perioperative outcomes, postoperative complication and follow-up data.

\section{Surgical Techniques:}

\section{Patient positioning and placement of the trocars}

The patient was placed in the supine position and general anesthesia was induced. A $12 \mathrm{~mm}$ trocar was inserted through the umbilical region by the open method, and carbon dioxide pneumoperitoneum was established. The additional four trocars were placed, including two $12 \mathrm{~mm}$ trocars in the right and left lower abdomen and two $5 \mathrm{~mm}$ trocars in the right and left upper abdomen.

\section{Laparoscopic intracorporeal Roux-en-Y gastrojejunostomy after distal gastrectomy}

Laparoscopic mobilization of the stomach and lymph node dissection were performed in a conventional manner. The vagus nerve was not preserved. The duodenum was divided distal to the pylorus with an endoscopic linear stapler (Endo-GIA 60-3, Covidien), then the stomach was divided with two endoscopic linear staplers. Usually one-third or one-fifth of the stomach was preserved. The specimen was removed 
through an extended $4 \mathrm{~cm}$ incision in the umbilical port. The jejunum, $20 \mathrm{~cm}$ distal to the ligament of Treitz, was prepared for a Roux limb, and the mesentery of this jejunum was divided for a distance of 8 $\mathrm{cm}$. The prepared jejunum then was divided with an endoscopic linear stapler to ensure a tension freegastrojejunostomy. A side-side jejunojejunostomy was fashioned $30 \mathrm{~cm}$ distal to the planed gastrojejunostomy using endoscopic linear staplers under direct vision through the umbilical incision. The jejunojejunostomy defect was closed with nonabsorbable sutures in an intermittent fashion. Pneumoperitoneum was rebuilt and the jejunal limb was brought to the gastric remnant through an antecolic route. A right-oriented Roux limb was created such that the cut end of the jejunal limb faced the greater curvature of the gastric remnant(Fig.b). The jejunal limb was anastomosed to the greater curvature of the stomach side-to-side with an endoscopic stapler, then the site of entry for the linear stapler was closed using a running hand sewn suture(Fig.c).

\section{Fixation of Roux Limb and Duodenal Stump}

We embedded the duodenal stump with a seromuscular suture and then fixed the alimentary limb onto the duodenal stump in a proper radian using non-absobable sutures(Fig.d,Fig.e). No intention was made to close the Petersen's defect in any of the patients during the surgery.

\section{Results}

Thirty-one consecutive patients underwent LDG with antecolic R-Y reconstruction for gastric cancer without intraoperative complications. Clinical characteristics and surgical outcomes are in Table 1. Two patients experienced pneumonia and pancreatitis after surgery, respectively. All patients were discharged with satisfactory recovery and were evaluated postoperatively by routine abdominal computed tomography scan and endoscopy as part of follow-up. The median follow-up period was 32.5 months (range 25-47 months). None of the patients had complications related to internal hernia and Roux stasis syndrome during the follow-up period.

\section{Discussion}

Herein we report our method to reduce post-operative Peterson's hernia and Roux stasis syndrome in patients undergoing LDG. There are reports that a laparoscopic approach, non-closure of mesenteric defects, and low BMI are independent risk factors for internal hernia by multivariate analysis[5.13.14.15]. One study reported a higher incidence of internal hernia after single-port surgery compared to multi-port surgery, resulting from the relatively difficult manipulation of laparoscopic instruments in single-port surgery[15].

The rate of Petersen's hernia which is a type of internal hernia, with the laparoscopic ante-colic R-Y reconstruction approach without closure of Petersen's defect, was reportedly $2.6 \%$ to $4.75 \%$ and generally was thought to be higher than with open approach [6.8.16]. One explanation is that laparoscopic surgery is less likely to form adhesions and promotes earlier intestinal peristalsis than laparotomy,which leads to 
a higher incidence of internal hernia [17]. Although all defects are closed at the time of the initial surgery, internal hernia still occurs in some cases[7.16]. Previous studies speculated that the large decrease in mesenteric fat after distal gastrectomy might lead to the reopening of the defect despite an initially complete suture [18.19.20]. An early diagnosis of internal hernia is a challenge for most surgeons because of atypical clinical symptoms and lack of sensitive imaging techniques[7]. Furthermore, the internal hernia may be delayed, so it is necessary to develop an effective technique to prevent an internal hernia during the surgery.

Closure of all the mesenteric defects with non-absorbable suture in a running fashion was considered the most effective method for preventing internal hernia[6.17.21], however, a tight closure of the Petersen's defect from the root of the mesentery of the Roux limb and transverse mesocolon to the transverse colon is difficult with the laparoscopic view. Unlike with laparoscopic gastric bypass, subtotal resection of the stomach with lymph node dissection widens the space behind the Roux limb in the ante-colic Roux-Y reconstruction, which may often result in a Petersen's hernia. As reported by Hirahara et al [17] one way to minimize the defect is to place the residual greater omentum in the defect between the Roux limb and the transverse mesocolon. Although this technique can prevent Petersen's hernia, it may not be suitable for patients undergoing total resection of the omentum.

The boundaries of Petersen's defect are defined as the transverse mesocolon, the mesentery of the Roux limb and the retroperitoneum [22]. In this approach to reducing the likelihood of Peterson's hernia, we initially fix the alimentary limb onto the duodenal stump with a proper radian and tension, then change the anatomy of Petersen's defect and narrow the space behind the Roux limb. This procedure decreases the mobility of alimentary limb and makes the mesentery of the alimentary limb and the transverse mesocolon stick tightly. Thus, this technique eliminates the narrowed Petersen's defect through rapid formation of adhesions behind Roux limb. Two large retrospective studies of internal hernia reported that the median interval time after gastrectomy for hernia formation was from 15 months to 20.9 months[13.15]. None of the patients developed internal hernias by at least two years of follow-up in this study. To some extent, the follow-up data supports our small change about the Roux limb in surgical technique. Compared with the many closure stitches needed to repair Petersen's defect in a relatively poor exposure, our reported procedure does not require mesentery stitches, is time-saving and is simple and effective.

In addition to reducing reflux, R-Y reconstruction potentially prevents the obstruction of the gastrointestinal anastomosis caused by recurrences around the pylorus and has a low incidence of anastomotic leak [23]. However, the rate of Roux stasis syndrome is higher in R-Y reconstruction than in Billroth $\otimes$ reconstruction [24.25], which had been reported to range from $10 \%$ to $30 \%$ [12]. Many factors have been inferred as the cause of Roux stasis syndrome, including a relatively large gastric remnant [26], an ectopic pacemaker from the Roux limb [27], a long length of Roux limb [28] and vagotomy [29]. Improved surgical reconstruction methods are used to reduce the rate of Roux stasis syndrome. Uncut R$\mathrm{Y}$ reconstruction is considered an effective method to reduce the incidence of Roux stasis syndrome [30]. Motoyama et al [31] reported that Roux stasis syndrome did not occurr in 105 patients who underwent $\beta$ - 
Shaped R-Y reconstruction with a Roux limb length of approximately $25-30 \mathrm{~cm}$ after LDG. Mimae et al [32] suggested that end-to-end anastomosis in gastrojejunostomy was superior to end-to-side anastomosis at preventing Roux stasis syndrome.

In addition, surgical technical factors with the Roux limb have been taken into account for the cause of Roux stasis syndrome. George [33] speculated that one of the causes for Roux stasis syndrome is partial obstruction near or at the gastrointestinal anastomosis, but without stomal stenosis. This author analyzed the types of partial obstruction and found that they were related to postoperative adhesions or a kinked loop around the gastrointestinal anastomosis as a result of non-standard surgical techniques. Masui et al [12] also suggested that the adhesion between the Roux limb and the suture of the gastric remnant, which was produced a strong bend of the Roux limb to the lesser curvature of gastric remnant, could be a cause of Roux stasis syndrome. To prevent this, we fix the alimentary limb onto the duodenal stump with appropriate tension, which ensures a fluent direction of the gastrointestinal anastomosis by avoiding the angle after the gastroenterostomy. Moreover, through this technique the alimentary limb lies to the right of the ligament of Treitz and does not cross the proximal jejunum (biliopancreatic limb), which allows digestive juices and food to pass smoothly into the distal small intestine.

\section{Conclusions}

Here we report a case series of a simple and effective method for preventing Petersen's hernia and Roux stasis syndrome following R-Y reconstruction. Although Petersen's hernia is a rare complication of R-Y reconstruction, it is difficult to diagnose and may cause serious postoperative complications that require additional surgery and may even result in death [13.15]. Roux stasis syndrome is also widely reported and results in poor post-operative nutritional status [24.25.28]. Furthermore, with our described technique, no patients had complications related to internal hernia and Roux stasis syndrome. This technique is a simple and effective method to decrease the incidence of internal hernia and Roux stasis syndrome.

\section{Abbreviations}

R-Y: Roux-en-Y

LDG: Laparoscopic Distal Gastrectomy

BMl: Body Mass Index

\section{Declarations}

\section{Acknowledgements}

Not applicable 


\section{Funding}

No financial support was obtained for this study

\section{Authors' contributions}

Tetsu Fukunaga, Jian-zhong Wu and Shinichi Oka designed the research; Sanae Kaji, Jian-zhong Wu, Shun Zhang and Yukinori Yube collected the clinical data; Satoshi Kanda,Yukinori Yube and Hiroyuki Egawa analyzed the data; Jian-zhong Wu and Hajime Orita wrote the paper; Tetsu Fukunaga corrected and approved the manuscript

\section{Corresponding author}

Correspondence to: Tetsu Fukunaga

\section{Author details}

${ }^{1}$ Department of Gastroenterology and Minimally Invasive Surgery,Juntendo University School of Medicine, Juntendo University, Tokyo, Japan; ${ }^{2}$ Department of Gastroenterology Surgery, Suzhou Ninth People's Hospital, Nantong University, Suzhou, China; ${ }^{3}$ Department of Surgery, Tongji University, Shanghai, China.

\section{Availability of data and materials}

The datasets generated during and/or analyzed during the current study are available from the corresponding author on request

\section{Competing interests}

The authors declare that they have no conflict of interest.

\section{Ethical approval and consent to participate}

Written informed consent was obtained from all individual participants included in the study, and the use of anonymized patient data was approved by the Ethics Committee of Juntendo University School of Medicine.

\section{References}


1. Thuy B. Tran, David J. Worhunsky, Malcolm H. Squires, et al. To Roux or not to Roux: a comparison between Roux-en-Y and Billroth II reconstruction following partial gastrectomy for gastric cancer.Gastric Cancer. 2016;19:994-1001

2. Kojima K, Yamada H, Inokuchi M, et al. Acomparison of Roux-en-Y and Billroth-I reconstruction after laparoscopy-assisted distal gastrectomy. Ann Surg. 2008;247:962-967

3. Ishikawa M, Kitayama J, Kaizaki S, et al. Prospective randomized trial comparing Billroth I and Rouxen-Y procedures after distal gastrectomy for gastric carcinoma. World J Surg. 2005;29:1415-1420

4. Imamura $\mathrm{H}$, Takiguchi S, Yamamoto K, et al. Morbidity and mortality results from a prospective randomized controlled trial comparing Billroth I and Roux-en-Y reconstructive procedures after distal gastrectomy for gastric cancer. World J Surg. 2012; 36: 632-637

5. Miyagaki H, Takiguchi S, Kurokawa Y, et al. Recent trend of internal hernia occurrence after gastrectomy for gastric cancer. World J Surg. 2012;36:851-857

6. Kazuyuki Kojima,Mlkito Inokuchi,Keiji Kato,et al. Petersen's hernia after laparoscopic distal gastrectomy with Roux-en-Y reconstruction for gastric cancer.Gastric Cancer. 2014;17:146-151

7. Steele KE,Prokopowicz GP,Magnuson T,et al. Laparoscopic antecolic Roux-en-Y gastric bypass with closure of internal defects leads to fewer internal hernias than the retrocolic approach.Surg Endosc. 2008;22:2056-2061

8. Quebbemann B, Dallal R. The orientation of the antecolic Roux limb markedly affects the incidence of internal hernias after laparoscopic gastric bypass. Obes Surg. 2005;15:766-770

9. Fukuhara K, Osugi $\mathrm{H}$, Takada $\mathrm{N}$, et al. Reconstructive procedure after distal gastrectomy for gastric cancer that best prevents duodenogastroesophageal reflux. World J Surg. 2002;26: 1452-1457

10. Shinoto K, Ochiai T, Suzuki T, et al. Effectiveness of Roux-en-Y reconstruction after distal gastrectomy based on an assessment of biliary kinetics. Surg Today. 2003; 33: 169-177

11. Bar-Natan M, Larson GM, Stephens G, et al. Delayed gastric emptying after gastric surgery. Am J Surg. 1996;172(1):24-28

12. Masui $T$, Kubora $T$, Nakanishi $Y$, et al. The flow angle beneath the gastrojejunostomy predicts delayed gastric emptying in Roux-en-Y reconstruction after distal gastrectomy. Gastric Cancer. $2012 ; 15$ : 281-286

13. Won Ho Han,Bang Wool Eom,Hong Man Yoon,et al. Clinical characteristics and surgical outcomes of internal hernia after gastrectomy in gastric cancer patients: retrospective case control study.Surgical Endoscopy. 2019.33(9):2873-2879

14. Kelly KJ, Allen PJ, Brennan MF, et al. Internal hernia after gastrectomy for cancer with Roux-Y reconstruction. Surgery. 2013;154(2):305-311

15. Kyong Min Kang,Yo Seok Cho,Sa-Hong Min,et al. Internal hernia after gastrectomy for gastric cancer in minimally invasive surgery era.Gastric Cancer. 2019.22(5):1009-1015

16. Hideyo Kimura , Mikimasa Ishikawa, Toshinaga Nabae, et al. Internal hernia after laparoscopicgastrectomy with Roux-en-Y reconstruction for gastric cancer.Asian Journal of Surgery. 
2017;40:203-209

17. Hirahara N, Matsubara T, Hayashi H, et al . Easy and secure closure of Petersen's defect after laparoscopic distal gastrectomy with Roux-en-Y reconstruction. J Laparoendosc Adv Surg Tech A. 2015;25:55-59

18. Hope WW, Sing RF, Chen AY, et al. Failure of mesenteric defect closure after Roux-en-Y gastric bypass. JSLS. 2010;14:213-216

19. Miyato H, Kitayama J, Hidemura A, et al . Vagus nerve preservation selectively restores visceral fat volume in patients with early gastric cancer who underwent gastrectomy. J Surg Res. 2012;173:6067

20. Papasavas PK, Caushaj PF, McCormick JT, et al. Laparoscopic management of complications following laparoscopic Roux-en-Y gastric bypass for morbid obesity. Obes Surg. 2002; 12:559-563

21. Higa KD, Ho T, Boone KB. Internal hernias after laparoscopic Roux-en Y gastric bypass: incidence, treatment, and prevention. Obes Surg. 2003;13:350-354

22. Rogers AM, lonescu AM, Pauli EM, et al . When is a Petersen's hernia not a Petersen's hernia. J Am Coll Surg. 2008;207:121-124

23. Hoya $\mathrm{Y}$, Mitsumori $\mathrm{N}$, Yanaga $\mathrm{K}$. The advantages and disadvantages of a Roux-en-Y reconstruction after a distal gastrectomy for gastric cancer. Surg Today. 2009;39(8):647-651

24. Mathias JR, Fernandez A, Sninsky CA, et al. Nausea, vomiting, and abdominal pain after Roux-en-Y anastomosis: motility of the jejunal limb. Gastroenterology. 1985; 88:101-107

25. Miedema BW, Kelly KA, Camilleri M, et al. Human gastric and jejunal transit and motility after Roux gastrojejunostomy. Gastroenterology. 1992;103:1133-1143

26. Cozzaglio L, Coladonato M, Dagrada CT, et al. Does the Roux-en-Y-stasis syndrome still exist?. Chir Ital. 2005;57(1):27-34

27. van der Mijle HC, Kleibeuker JH, Limburg AJ, et al. Manometric and scintigraphic studies of the relation between motility disturbances in the Roux limb and the Roux-en-Y syndrome. Am J Surg. 1993; 166(1):11-17

28. Gustavsson S, llstrup DM, Morrison P, et al. Roux-Y stasis syndrome after gastrectomy. Am J Surg. 1988;155:490-494

29. Mathias JR, Khanna R, Nealon WH, et al. Roux-limb motility after total gastrectomy and Roux-en-Y anastomosis in patients with Zollinger-Ellison syndrome.Dig Dis Sci. 1992; 37(4):545-550

30. Ji Yeon Park, Yong Jin Kim. Uncut Roux-en-Y reconstruction after laparoscopic distal gastrectomy can be a favorable method in terms of gastritis, bile reflux, and gastric residue. J Gastric Cancer. 2014;14(4):229-237

31. Kazuo Motoyama,Kazuyuki Kojima,Mikiko Hayashi,et al. $\beta$-Shaped intracorporeal Roux-en-Y reconstruction after totally laparoscopic distal gastrectomy.Gastric Cancer. 2014;17:588-593

32. Mimae T, Ninomiya $M$, Nishizaki $M$, et al. Clinical study of Roux stasis syndrome after distal gastrectomy. Jpn Soc Gastroenterol Surg. 2008;41(8):1551-1556. 
33. George F,Gowen. Delayed Gastric Emptying After Roux-en-Y due to Four Types of Partial Obstruction.Ann.Surg. 1992;215:363-367

\section{Table}

Table 1. Clinical characteristics (including treatment details) of patients and surgical outcomes $(n=$ 31)

Sex

$$
\text { Male/Female }
$$

$19 / 12$

Age Mean (y) $66.2 \pm 13.0$

Body mass index $\mathrm{kg} / \mathrm{m}^{2}$ $21.5 \pm 2.7$

Extent of lymph node dissection

$$
\text { D1+/D2 }
$$

$16 / 15$

Pathological tumor stage

IA/IB/IIA/IIB/IIIA/IIIB

Operation time

Intraoperative blood loss

Postoperative hospital stay

Conversion to open surgery

Postoperative complication

Anastomotic leak or stenosis

Anastomotic bleeding

Roux stasis syndrome

Internal hernia

Pancreatitis

$15 / 1 / 2 / 6 / 6 / 1$

Pneumonia

Reoperation

Readmission

$\mathrm{min}$
$\mathrm{ml}$
days
$(\%)$
$(\%)$
$308.0 \pm 84.6$

$70.1 \pm 76.0$

$13.5 \pm 2$

$0(0)$

2(6.5)

$0(0)$

$0(0)$

$0(0)$

0(0)

1(3.2)

1(3.2)

(\%)

(\%)

$0(0)$

$0(0)$

Number of patients is shown unless otherwise indicated. Values are shown as mean \pm SD. Body Mass Index $=$ body weight $/$ height $^{2}\left(\mathrm{~kg} / \mathrm{m}^{2}\right)$.

\section{Figures}




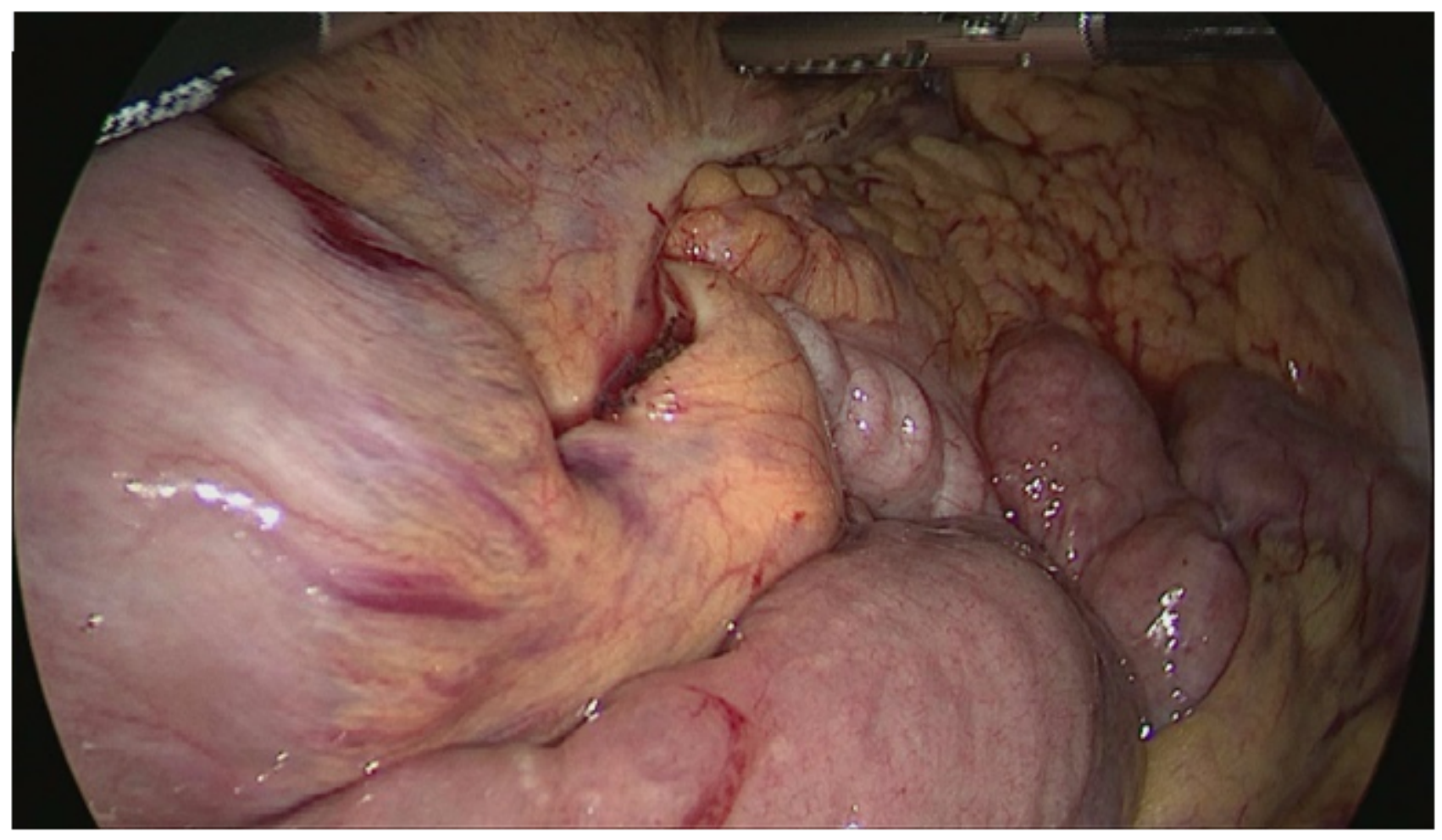

Figure 1

Petersen's defect (white arrow)

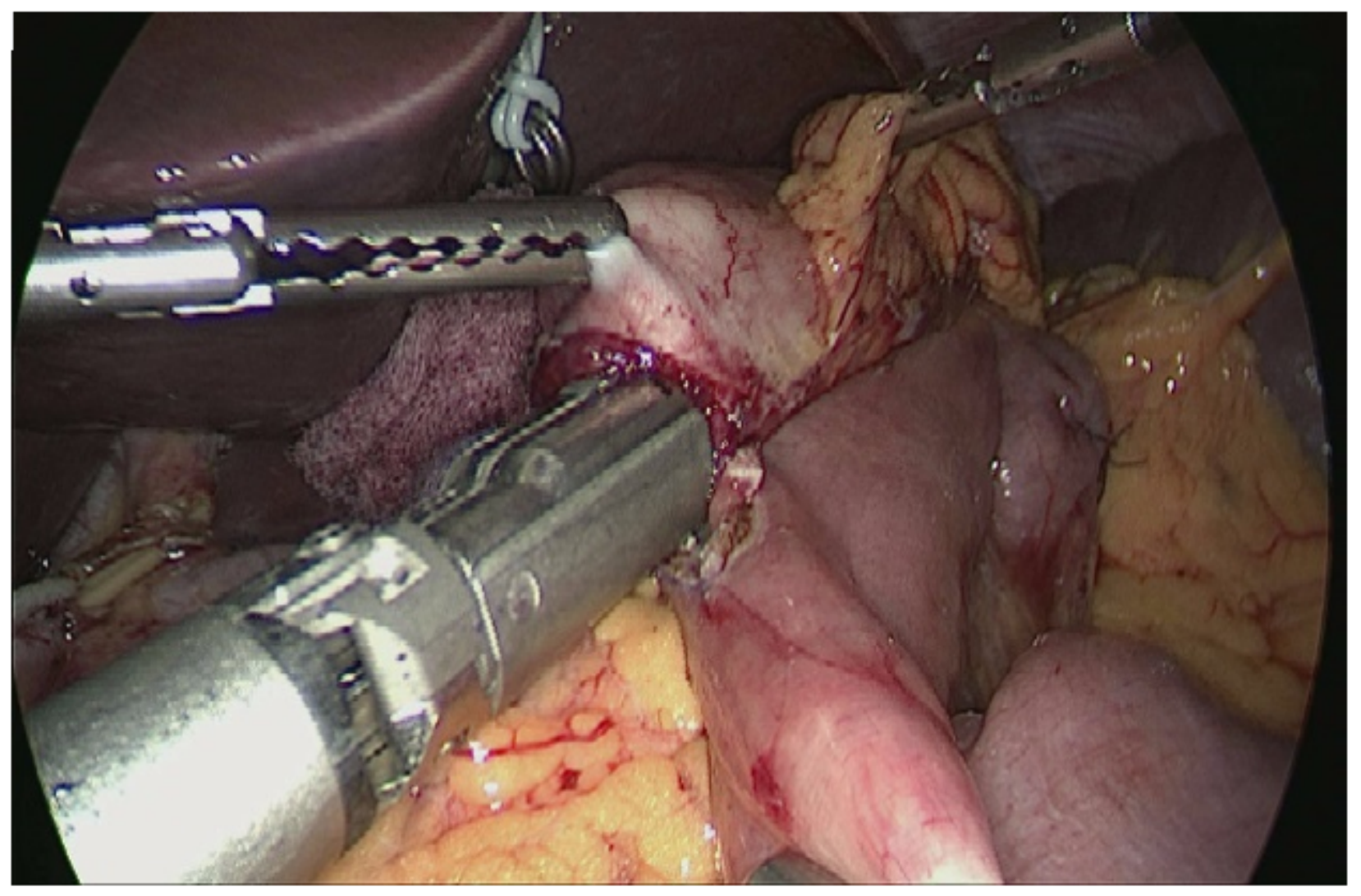

Figure 2 
the cut end of the jejunal limb faced the greater curvature

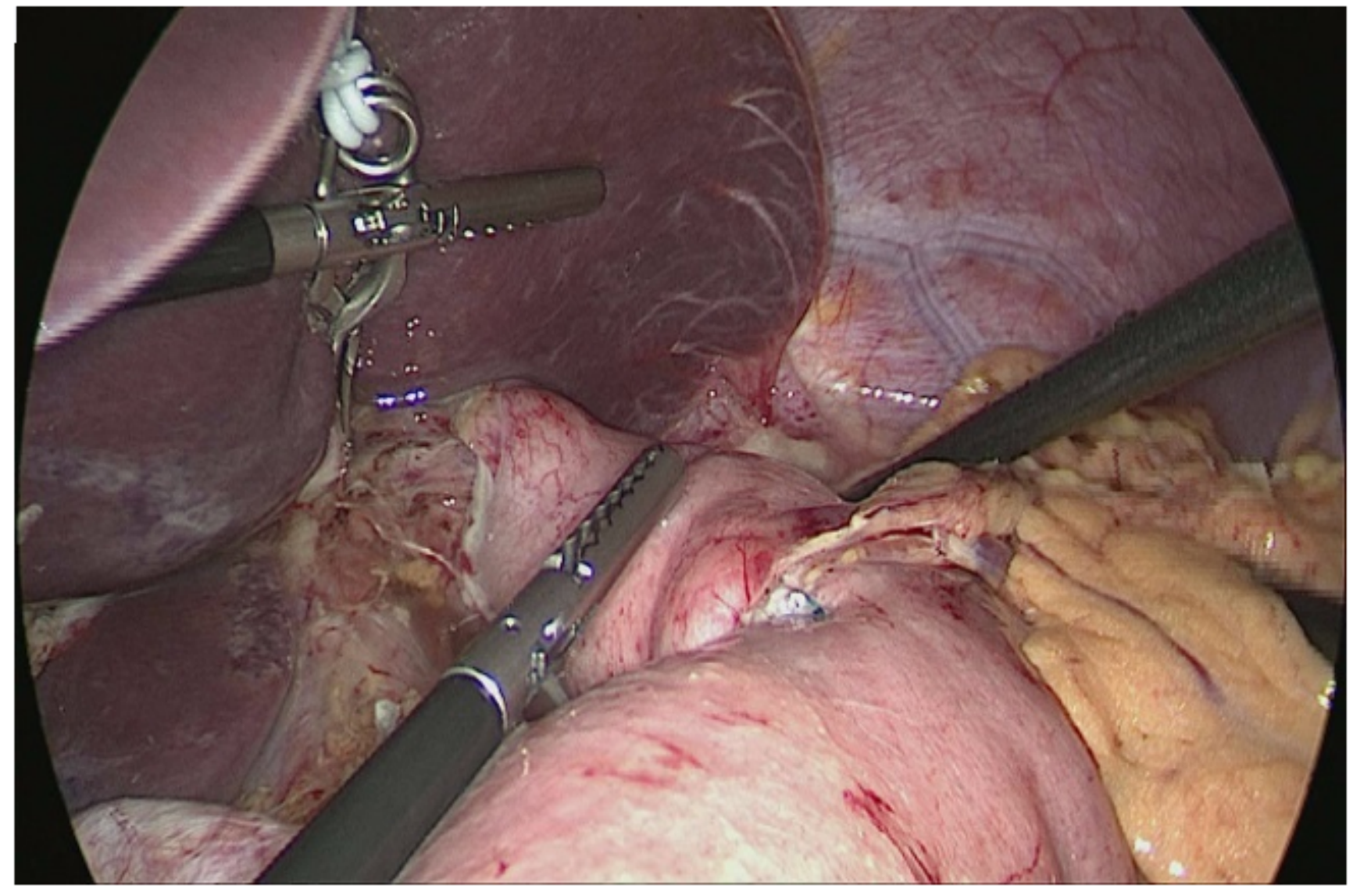

\section{Figure 3}

the site of entry was closed using a running handsewn suture 


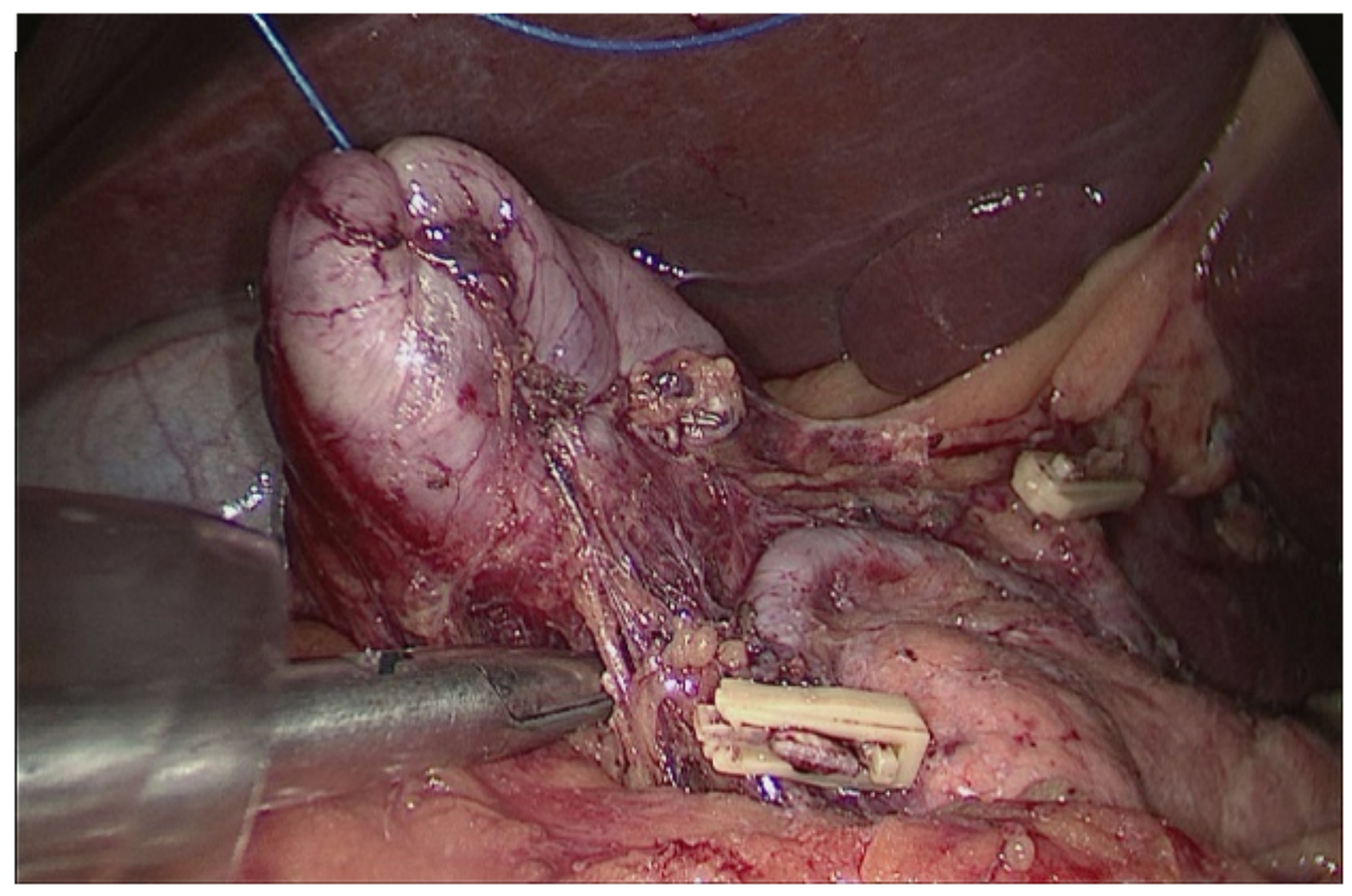

Figure 4

the duodenal stump was embedded with seromuscular suture

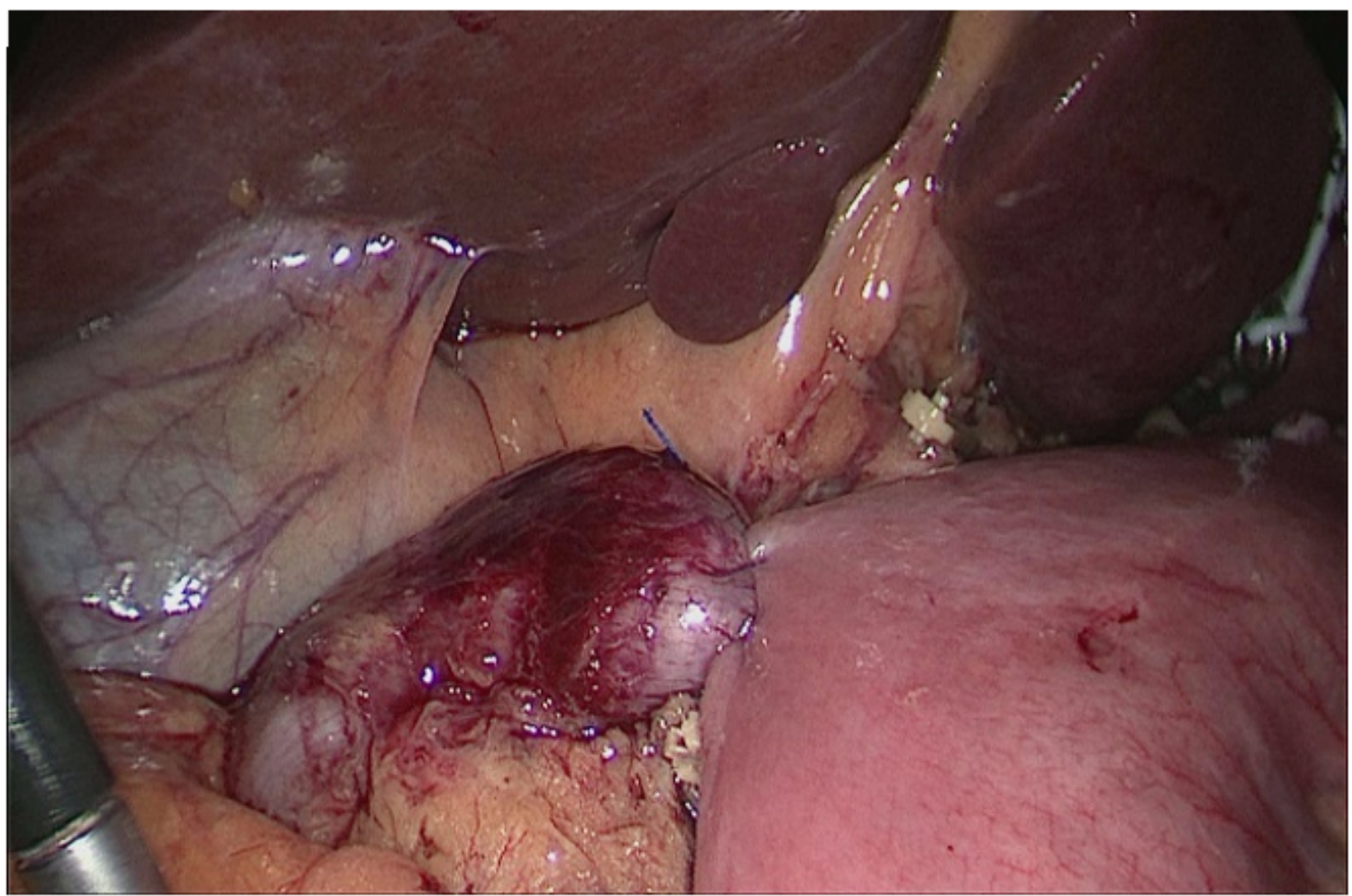


Figure 5

the alimentary limb was fixed on the duodenal stump 